\title{
RACSAM
}

Rev. R. Acad. Cien. Serie A. Mat.

VoL. 103 (1), 2009, pp. 55-74

Análisis Matemático / Mathematical Analysis

\section{On the ultradistributions of Beurling type}

\author{
Manuel Valdivia
}

\begin{abstract}
Let $\Omega$ be a nonempty open set of the $k$-dimensional euclidean space $\mathbb{R}^{k}$. In this paper, we show that if $S$ is an ultradistribution in $\Omega$, belonging to a class of Beurling type stable under differential operators, then $S$ can be represented in the form $\sum_{\alpha \in \mathbb{N}_{0}^{k}} D^{\alpha} f_{\alpha}$, where $f_{\alpha}$ is a complex function defined in $\Omega$ which is Lebesgue measurable and essentially bounded in each compact subset of $\Omega$. Other structure results on certain ultradistributions are obtained, too.
\end{abstract}

\section{Ultradistribuciones de tipo Beurling}

Resumen. Sea $\Omega$ un conjunto abierto no vacío del espacio euclídeo . En este artículo se demuestra que si $\mathrm{S}$ es una ultradistribución en $\Omega$, perteneciente a una clase de tipo Beurling que sea estable frente a operadores diferenciales, entonces $\mathrm{S}$ se puede representar en la forma $\sum_{\alpha \in \mathbb{N}_{0}^{k}} D^{\alpha} f_{\alpha}$, donde $f_{\alpha}$ es una función compleja definida en $\Omega$ que es Lebesgue medible y esencialmente acotada en cada subconjunto compacto de $\Omega$. También se obtienen otros resultados de estructura de ciertas ultradistribuciones.

\section{Introduction and notation}

Throughout this paper all linear spaces are assumed to be defined over the field $\mathbb{C}$ of complex numbers. We write $\mathbb{N}$ for the set of positive integers and by $\mathbb{N}_{0}$ we mean the set of nonnegative integers. If $E$ is a locally convex space, $E^{\prime}$ will be its topological dual and $\langle\cdot, \cdot\rangle$ will denote the standard duality between $E$ and $E^{\prime}$. Given a Banach space $X, B(X)$ denotes its closed unit ball and $X^{*}$ is the Banach space conjugate of $X$. Given a positive integer $k$, if $\alpha:=\left(\alpha_{1}, \alpha_{2}, \ldots, \alpha_{k}\right)$ is a multiindex of order $k$, i.e., an element of $\mathbb{N}_{0}^{k}$, we put $|\alpha|$ for its length, that is, $|\alpha|=\alpha_{1}+\alpha_{2}+\cdots+\alpha_{k}$, and $\alpha !:=\alpha_{1} ! \alpha_{2} ! \cdots \alpha_{k} !$.

Given a complex function $f$ defined in the points $x=\left(x_{1}, x_{2}, \ldots, x_{k}\right)$ of an open subset $O$ of the $k$-dimensional euclidean space $\mathbb{R}^{k}$, and being infinitely differentiable, we write

$$
D^{\alpha} f(x):=\frac{\partial^{|\alpha|} f(x)}{\partial x_{1}^{\alpha_{1}} \partial x_{2}^{\alpha_{2}} \ldots \partial x_{k}^{\alpha_{k}}}, \quad x \in O, \quad \alpha \in \mathbb{N}_{0}^{k} .
$$

We consider a sequence $M_{0}, M_{1}, \ldots, M_{n}, \ldots$ of positive numbers satisfying the following conditions:

1. $M_{0}=1$.

2. Logarithmic convexity:

$$
M_{n}^{2} \leq M_{n-1} M_{n+1}, \quad n \in \mathbb{N} .
$$

Presentado por / Submitted by Darío Maravall Casesnoves.

Recibido / Received: 20 de febrero de 2008 Aceptado / Accepted: 4 de marzo de 2009.

Palabras clave / Keywords: Beurling type, differential operators, ultradistributions.

Mathematics Subject Classifications: 46F05.

(C) 2009 Real Academia de Ciencias, España. 
3. Non-quasi-analyticity:

$$
\sum_{n=1}^{\infty} \frac{M_{n-1}}{M_{n}}<\infty
$$

Let us take a nonempty open set $\Omega$ in $\mathbb{R}^{k}$. A complex function $f$, defined and infinitely differentiable in $\Omega$, is said to be ultradifferentiable of class $\left(M_{n}\right)$ whenever, given $h>0$ and a compact subset $K$ of $\Omega$, there is $C>0$ such that

$$
\left|D^{\alpha} f(x)\right| \leq C h^{|\alpha|} M_{|\alpha|}, \quad x \in K, \quad \alpha \in \mathbb{N}_{0}^{k} .
$$

We put $\mathcal{E}^{\left(M_{n}\right)}(\Omega)$ to denote the linear space over $\mathbb{C}$ formed by all the ultradifferentiable functions of class $\left(M_{n}\right)$ defined in $\Omega$, with the ordinary topology, [2]. By $\mathcal{D}^{\left(M_{n}\right)}(\Omega)$ we denote the linear subspace of $\mathcal{E}^{\left(M_{n}\right)}(\Omega)$ formed by those functions which have compact support.

We now choose a fundamental sequence of compact subsets of $\Omega$ :

$$
K_{1} \subset K_{2} \subset \cdots \subset K_{m} \cdots
$$

If $K$ is an arbitrary compact subset of $\Omega$, we use $\mathcal{D}^{\left(M_{n}\right)}(K)$ to denote the subspace of $\mathcal{E}^{\left(M_{n}\right)}(\Omega)$ formed by those functions which have their support in $K$. We then have that

$$
\mathcal{D}^{\left(M_{n}\right)}(\Omega)=\bigcup_{m=1}^{\infty} \mathcal{D}^{\left(M_{n}\right)}\left(K_{m}\right) .
$$

We consider $\mathcal{D}^{\left(M_{n}\right)}(\Omega)$ as the inductive limit of the sequence $\left(\mathcal{D}^{\left(M_{n}\right)}\left(K_{m}\right)\right)$ of Fréchet spaces. The elements of the topological dual $\mathcal{D}^{\left(M_{n}\right)^{\prime}}(\Omega)$ of $\mathcal{D}^{\left(M_{n}\right)}(\Omega)$ are called ultradistributions of Beurling type in $\Omega$. We assume that $\mathcal{D}^{\left(M_{n}\right)^{\prime}}(\Omega)$ has its strong topology.

By $\mathcal{K}(\Omega)$ we mean the linear space over $\mathbb{C}$ of the complex functions defined in $\Omega$ which are continuous and have compact support. If $K$ is any compact subset of $\Omega, \mathcal{K}(K)$ is the subspace of $\mathcal{K}(\Omega)$ formed by the functions with support contained in $K$. If $f$ is in $\mathcal{K}(K)$, we put

$$
|f|_{\infty}:=\sup _{x \in \Omega}|f(x)|,
$$

and assume that $\mathcal{K}(K)$ is endowed with the norm $|\cdot|_{\infty}$.

We consider $\mathcal{K}(\Omega)$ as the inductive limit of the sequence $\left(\mathcal{K}\left(K_{m}\right)\right)$ of Banach spaces. A Radon measure in $\Omega$ is an element of the topological dual $\mathcal{K}^{\prime}(\Omega)$ of $\mathcal{K}(\Omega)$. Given a Radon measure $u$ in $\Omega$ and a compact subset $K$ of $\Omega$, we put $\|u\|(K)$ for the norm of the restriction of $u$ to the Banach space $\mathcal{K}(K)$.

In [2, p. 76], a structure theorem for ultradistributions of Beurling type in $\Omega$ is given. It can be stated as follows:

Result a) If $S$ is an element of $\mathcal{D}^{\left(M_{n}\right)^{\prime}}(\Omega)$ and $G$ is an open subset of $\Omega$ which is relatively compact, for each $\alpha \in \mathbb{N}_{0}^{k}$, we may find an element $v_{\alpha}$ in the conjugate of the Banach space $\mathcal{K}(\bar{G})$, whose norm we represent by $\left\|v_{\alpha}\right\|$, such that, for some $h>0$,

$$
\sup _{\alpha \in \mathbb{N}_{0}^{k}} h^{|\alpha|} M_{|\alpha|}\left\|v_{\alpha}\right\|<\infty
$$

and

$$
S_{\mid G}=\sum_{\alpha \in \mathbb{N}_{0}^{k}} D^{\alpha} v_{\alpha} .
$$

The above result is of local character, for the elements $v_{\alpha}, \alpha \in \mathbb{N}_{0}^{k}$, depend on $G$. In [4], we give a structure theorem of global character for the ultradistributions of Beurling type in $\Omega$. This theorem contains result a) as a particular case and can be stated as follows: 
Result b) If $S$ is an element of $\mathcal{D}^{\left(M_{n}\right)^{\prime}}(\Omega)$, then there is a family $\left(u_{\alpha}: \alpha \in \mathbb{N}_{0}^{k}\right)$ of Radon measures in $\Omega$ such that

$$
\langle\varphi, S\rangle=\sum_{\alpha \in \mathbb{N}_{0}^{k}}\left\langle D^{\alpha} \varphi, u_{\alpha}\right\rangle, \quad \varphi \in \mathcal{D}^{\left(M_{n}\right)}(\Omega),
$$

where the series converges absolutely and uniformly on every bounded subset of $\mathcal{D}^{\left(M_{n}\right)}(\Omega)$. Also, given a compact subset $K$ of $\Omega$, there is $h>0$ such that

$$
\sup _{\alpha \in \mathbb{N}_{0}^{k}} h^{|\alpha|} M_{|\alpha|}\left\|u_{\alpha}\right\|(K)<\infty .
$$

We now put $\mathcal{L}_{\text {loc }}^{\infty}(\Omega)$ for the linear space over $\mathbb{C}$ formed by the complex functions defined in $\Omega$, which are Lebesgue-measurable and essentially bounded in every compact subset of $\Omega$. The elements of this space are considered as Radon measures on $\Omega$ in the usual way. If $f$ is in $\mathcal{L}_{\text {loc }}^{\infty}(\Omega)$ and $K$ is a compact subset of $\Omega$, we write $|f|_{K, \infty}$ for the essential supremum of $|f|$ in $K$.

We say that the sequence $M_{0}, M_{1}, \ldots, M_{n}, \ldots$ satisfies the stability condition for differential operators provided there are $A>0$ and $h>0$ such that

$$
M_{n+1} \leq A h^{n} M_{n}, \quad n \in \mathbb{N}_{0} .
$$

In this paper, we give a structure theorem for ultradistributions of Beurling type in $\Omega$, which contains the following result as a particular case:

Result c) If $M_{0}, M_{1}, \ldots, M_{n}, \ldots$ satisfies condition (1) and $S$ is an element of $\mathcal{D}^{\left(M_{n}\right)^{\prime}}(\Omega)$, then there is a family $\left(f_{\alpha}: \alpha \in \mathbb{N}_{0}^{k}\right)$ of elements of $\mathcal{L}_{\mathrm{loc}}^{\infty}(\Omega)$ such that, given an arbitrary compact subset $K$ of $\Omega$, there is $h>0$ with

$$
\sup _{\alpha \in \mathbb{N}_{0}^{k}} h^{|\alpha|} M_{|\alpha|}\left|f_{\alpha}\right|_{K, \infty}<\infty
$$

and

$$
S=\sum_{\alpha \in \mathbb{N}_{0}^{k}} D^{\alpha} f_{\alpha}
$$

\section{Basic constructions}

Let $X$ be a Banach space. We put $\|\cdot\|$ for the norm of $X$ and also for the norm of $X^{*}$. Given $r \in \mathbb{N}$ and $\alpha \in \mathbb{N}_{0}^{k}$, we put, for each $x \in X$,

$$
|x|_{r, \alpha}:=\frac{r^{|\alpha|}\|x\|}{M_{|\alpha|}} .
$$

We denote by $X_{r, \alpha}$ the linear space $X$ provided with the norm $|\cdot|_{r, \alpha}$. By $X_{r, \alpha}^{*}$ we mean the Banach space conjugate of $X_{r, \alpha}$ with $|\cdot|_{r, \alpha}$ as its norm. Clearly, if $u$ is in $X^{*}$, then

$$
|u|_{r, \alpha}=\frac{M_{|\alpha|}}{r^{|\alpha|}}\|u\| .
$$

We put $Z_{r}$ for the linear space over $\mathbb{C}$ of the families $\left(x_{\alpha}: \alpha \in \mathbb{N}_{0}^{k}\right)$ of elements of $X$, which we shall just denote by $\left(x_{\alpha}\right)$, such that

$$
\left\|\left(x_{\alpha}\right)\right\|_{r}:=\sup _{\alpha \in \mathbb{N}_{0}^{k}} \frac{r^{|\alpha|}\left\|x_{\alpha}\right\|}{M_{|\alpha|}}<\infty .
$$

We assume that $Z_{r}$ is provided with the norm $\|\cdot\|_{r}$ It then follows that $Z_{r} \supset Z_{r+1}$ and that the canonical injection from $Z_{r+1}$ into $Z_{r}$ is continuous.

We write $Z$ to denote the Fréchet space given by the projective limit of the sequence $\left(Z_{r}\right)$ of Banach spaces. We assume $Z^{\prime}$ endowed with the strong topology. 
Given $\beta$ in $\mathbb{N}_{0}^{k}$, we put $Z^{\beta}$ for the subspace of $Z$ whose elements $\left(x_{\alpha}\right)$ satisfy that $x_{\alpha}=0$ when $\alpha$ is distinct from $\beta$. We then have that $Z^{\beta}$ is topologically isomorphic to $X$ and, considering $Z^{\beta}$ as a subspace of $Z_{r}$, then it is isometric to $X_{r, \beta}$.

If $u$ is an arbitrary element of $Z^{\prime}$ and $r \in \mathbb{N}$, we put

$$
\|u\|_{(r)}:=\sup \left\{\left|\left\langle\left(x_{\alpha}\right), u\right\rangle\right|:\left(x_{\alpha}\right) \in B\left(Z_{r}\right) \cap Z\right\} .
$$

For each $u \in Z^{\prime}$ and each $\beta \in \mathbb{N}_{0}^{k}$, we identify, in the usual manner, the restriction of $u$ to $Z^{\beta}$ with an element $u_{\beta}$ of $X^{*}$.

If $\left(x_{\alpha}\right)$ is an element of $Z$ and $\beta$ is in $\mathbb{N}_{0}^{k}$, we write

$$
x_{\alpha}^{\beta}:= \begin{cases}x_{\beta}, & \text { if } \alpha=\beta, \\ 0, & \text { if } \alpha \neq \beta .\end{cases}
$$

Clearly, $\left(x_{\alpha}^{\beta}\right)$ belongs to $Z$ and, for each $r \in \mathbb{N}$,

$$
\left\|\left(x_{\alpha}^{\beta}\right)\right\|_{r} \leq\left\|\left(x_{\alpha}\right)\right\|_{r} .
$$

The next proposition unifies Proposition 1 and the Note in [4].

Proposition 1. If $M$ is a bounded subset of $Z^{\prime}$, then there is $r$ in $\mathbb{N}$ such that

$$
\sup _{\substack{\alpha \in \mathbb{N}_{0}^{k} \\ u \in M}} r^{-|\alpha|} M_{|\alpha|}|| u_{\alpha} \| \leq 1
$$

and

$$
\left\langle\left(x_{\alpha}\right), u\right\rangle=\sum_{\alpha \in \mathbb{N}_{0}^{k}}\left\langle x_{\alpha}, u_{\alpha}\right\rangle, \quad u \in M, \quad\left(x_{\alpha}\right) \in Z,
$$

where the series converges absolutely and uniformly when $u$ varies in $M$ and $\left(x_{\alpha}\right)$ varies in any given bounded subset of $Z$.

Proof. If $M^{\circ}$ is the polar set of $M$ in $Z$, we find $r \in \mathbb{N}$ such that $B\left(Z_{r}\right) \cap Z$ is contained in $M^{\circ}$. Then, for each $u \in M$, we have, if we fix $\beta \in \mathbb{N}_{0}^{k}$,

$$
\begin{aligned}
1 & \geq\|u\|_{(r)}=\sup \left\{\left|\left\langle\left(x_{\alpha}\right), u\right\rangle\right|:\left(x_{\alpha}\right) \in B\left(Z_{r}\right) \cap Z\right\} \\
& \geq \sup \left\{\left|\left\langle\left(x_{\alpha}^{\beta}\right), u\right\rangle\right|:\left(x_{\alpha}\right) \in B\left(Z_{r}\right) \cap Z\right\} \\
& =\sup \left\{\left|\left\langle\left(x_{\beta}\right), u_{\beta}\right\rangle\right|:\left|x_{\beta}\right|_{r, \beta} \leq 1\right\}=\left|u_{\beta}\right|_{r, \beta} \\
& =\frac{M_{|\beta|}}{r^{|\beta|}}\left\|u_{\beta}\right\|
\end{aligned}
$$

from where we deduce

$$
\sup _{\substack{\alpha \in \mathbb{N}_{0}^{k} \\ u \in M}} r^{-|\alpha|} M_{|\alpha|} \mid\left\|u_{\alpha}\right\| \leq 1 .
$$

We take $\left(x_{\alpha}\right)$ in $Z$ and we see that $\left(\left(x_{\alpha}^{\beta}\right): \beta \in \mathbb{N}_{0}^{k}\right)$ is summable in $Z$ to $\left(x_{\alpha}\right)$. Let $s, q$ be in $\mathbb{N}$. We then have

$$
\begin{aligned}
\left\|\left(x_{\alpha}\right)-\sum_{|\beta| \leq q}\left(x_{\alpha}^{\beta}\right)\right\|_{s} & =\sup _{|\alpha|>q} \frac{s^{|\alpha|}\left\|x_{\alpha}\right\|}{M_{|\alpha|}} \\
& =\sup _{|\alpha|>q} \frac{(2 s)^{|\alpha|}\left\|x_{\alpha}\right\|}{2^{|\alpha|} M_{|\alpha|}} \\
& \leq \frac{1}{2^{q}} \sup _{\alpha \in \mathbb{N}_{0}^{k}} \frac{(2 s)^{|\alpha|}\left\|x_{\alpha}\right\|}{M_{|\alpha|}} \\
& =\frac{1}{2^{q}}\left\|\left(x_{\alpha}\right)\right\|_{2 s}
\end{aligned}
$$


and the conclusion follows. From

$$
\left(x_{\alpha}\right)=\sum_{\beta \in \mathbb{N}_{0}^{k}}\left(x_{\alpha}^{\beta}\right)
$$

in $Z$, we obtain

$$
\left\langle\left(x_{\alpha}\right), u\right\rangle=\sum_{\beta \in \mathbb{N}_{0}^{k}}\left\langle\left(x_{\alpha}^{\beta}\right), u\right\rangle=\sum_{\beta \in \mathbb{N}_{0}^{k}}\left\langle x_{\beta}, u_{\beta}\right\rangle, \quad u \in Z^{\prime} .
$$

We consider now a bounded subset $B$ of $Z$. We find $b>0$ such that $B \subset b B\left(Z_{2 k r}\right)$. We choose arbitrary elements $\left(x_{\alpha}\right) \in B$ and $u \in M$. We fix $\beta \in \mathbb{N}_{0}^{k}$. Then

$$
\begin{aligned}
\left|\left\langle x_{\beta}, u_{\beta}\right\rangle\right| & \leq\left\|x_{\beta}\right\| \cdot\left\|u_{\beta}\right\| \\
& =\frac{(2 k r)^{|\beta|}\left\|x_{\beta}\right\|}{M_{|\beta|}} \cdot \frac{M_{|\beta|}\left\|u_{\beta}\right\|}{(2 k r)^{|\beta|}} \\
& \leq \frac{1}{(2 k)^{|\beta|}}\left\|\left(x_{\alpha}\right)\right\|_{2 k r} \sup _{\substack{\alpha \in \mathbb{N}_{0}^{k} \\
u \in M}} r^{-|\alpha|} M_{|\alpha|}\left\|u_{\alpha}\right\| \\
& \leq \frac{1}{(2 k)^{|\beta|}} b
\end{aligned}
$$

and, since

$$
\sum_{\beta \in \mathbb{N}_{0}^{k}} \frac{1}{(2 k)^{|\beta|}}=2
$$

the conclusion follows.

The following proposition may be found in [4].

Proposition 2. Let $\left\{v_{\alpha}: \alpha \in \mathbb{N}_{0}^{k}\right\}$ a family of elements of $X^{*}$ such that there is $h>0$ with

$$
\sup _{\alpha \in \mathbb{N}_{0}^{k}} h^{|\alpha|} M_{|\alpha|}\left\|v_{\alpha}\right\|<\infty .
$$

Then, there is a unique element $u \in Z^{\prime}$ such that $u_{\alpha}=v_{\alpha}, \alpha \in \mathbb{N}_{0}^{k}$.

\section{The space $\mathcal{D}_{\left(L^{p}\right)}^{\left(M_{n}\right)}(\Omega)$}

We put $L^{p}\left(\Omega\right.$ and $\mathcal{L}^{p}(\Omega), 1 \leq p \leq \infty$, for the classical Lebesgue spaces. If $f \in \tilde{f} \in \mathcal{L}^{p}(\Omega), 1 \leq p<\infty$, we write

$$
\|f\|_{p}=\|\tilde{f}\|_{p}=\left(\int_{\Omega}|f|^{p} \mathrm{~d} x\right)^{1 / p}
$$

and, if $f \in \tilde{f} \in L^{\infty}(\Omega)$, then

$$
\|f\|_{\infty}=\|\tilde{f}\|_{\infty}=\operatorname{supess}\{|f(x)|: x \in \Omega\} .
$$

$\mathcal{D}_{L^{p}}\left(\mathbb{R}^{k}\right), 1 \leq p<\infty$, is the classical L. Schwartz's space, [3, p. 199]. We put $\mathcal{B}_{L^{p}}(\Omega)$ for the linear space over $\mathbb{C}$ of the complex functions $f$ defined in $\Omega$ which are infinitely differentiable and such that $D^{\alpha} f$ is in $\mathcal{L}^{p}(\Omega), \alpha \in \mathbb{N}_{0}^{k}$. We assume that $\mathcal{B}_{L^{p}}(\Omega)$ is endowed with the metrizable locally convex topology such that a sequence $\left(f_{n}\right)$ in $\mathcal{B}_{L^{p}}(\Omega)$ converges to the origin if and only if $\left(\left\|D^{\alpha} f_{n}\right\|_{p}\right)$ converges to zero for each $\alpha \in \mathbb{N}_{0}^{k}$. We then have that $\mathcal{B}_{L^{p}}(\Omega)$ is a Fréchet space. Clearly, $\mathcal{B}_{L^{p}}\left(\mathbb{R}^{k}\right)$ coincides with $\mathcal{D}_{L^{p}}\left(\mathbb{R}^{k}\right)$. 
Given $r \in \mathbb{N}$ and $1 \leq p<\infty$, we put $\mathcal{B}_{L^{p}}^{\left(M_{n}\right), 1 / r}(\Omega)$ for the linear space over $\mathbb{C}$ of the functions $f \in \mathcal{B}_{L^{p}}(\Omega)$ which satisfy:

$$
\|f\|_{p, 1 / r}:=\sup _{\alpha \in \mathbb{N}_{0}^{k}} \frac{r^{|\alpha|}\left\|D^{\alpha} f\right\|_{p}}{M_{|\alpha|}}<\infty
$$

We assume $\mathcal{B}_{L^{p}}^{\left(M_{n}\right), 1 / r}(\Omega)$ provided with the norm $\|\cdot\|_{p, 1 / r}$. Given a Cauchy sequence $\left(f_{m}\right)$ in $\mathcal{B}_{L^{p}}^{\left(M_{n}\right), 1 / r}(\Omega)$, it is immediate that $\left(f_{m}\right)$ is a Cauchy sequence in $\mathcal{B}_{L^{p}}(\Omega)$ and hence it converges in this space to a function $f$. Given $\varepsilon>0$, there is a positive integer $m_{0}$ such that

$$
\left\|f_{m}-f_{s}\right\|_{p, 1 / r}<\varepsilon, \quad m, s \geq m_{0} .
$$

Then, for those values of $m$ and $s$, and for each $\alpha \in \mathbb{N}_{0}^{k}$, we have that

$$
\frac{r^{|\alpha|}\left\|D^{\alpha} f_{m}-D^{\alpha} f_{s}\right\|_{p}}{M_{|\alpha|}}<\varepsilon
$$

and therefore, for $m \geq m_{0}$,

$$
\frac{r^{|\alpha|}\left\|D^{\alpha} f_{m}-D^{\alpha} f\right\|_{p}}{M_{|\alpha|}} \leq \varepsilon
$$

from where we deduce that $f$ belongs to $\mathcal{B}_{L^{p}}^{\left(M_{n}\right), 1 / r}(\Omega)$ and that $\left\|f_{m}-f\right\|_{p, 1 / r} \leq \varepsilon, m \geq m_{0}$. Consequently, $\mathcal{B}_{L^{p}}^{\left(M_{n}\right), 1 / r}(\Omega)$ is a Banach space.

It is plain that $\mathcal{B}_{L^{p}}^{\left(M_{n}\right), \frac{1}{r+1}}(\Omega)$ is contained in $\mathcal{B}_{L^{p}}^{\left(M_{n}\right), 1 / r}(\Omega)$ and also that the canonical injection from $\mathcal{B}_{L^{p}}^{\left(M_{n}\right), \frac{1}{r+1}}(\Omega)$ into $\mathcal{B}_{L^{p}}^{\left(M_{n}\right), 1 / r}(\Omega)$ is continuous. We denote by $\mathcal{B}_{L^{p}}^{\left(M_{n}\right)}(\Omega)$ the projective limit of the sequence $\left(\mathcal{B}_{L^{p}}^{\left(M_{n}\right), 1 / r}(\Omega)\right)$ of Banach spaces. We assume that the topological dual $\mathcal{B}_{L^{p}}^{\left(M_{n}\right)^{\prime}}(\Omega)$ of $\mathcal{B}_{L^{p}}^{\left(M_{n}\right)}(\Omega)$ is endowed with the strong topology.

In this section we substitute the Banach space $X$ of the previous section by $L^{p}(\Omega)$. Then, every element of $Z_{r}$ is a family $\left(\tilde{f}_{\alpha}: \alpha \in \mathbb{N}_{0}^{k}\right)$ of elements of $L^{p}(\Omega)$ such that

$$
\left\|\left(\tilde{f}_{\alpha}\right)\right\|_{r}=\sup _{\alpha \in \mathbb{N}_{0}^{k}} \frac{r^{|\alpha|}\left\|\tilde{f}_{\alpha}\right\|_{p}}{M_{|\alpha|}}<\infty .
$$

If $f$ belongs to $\mathcal{B}_{L^{p}}^{\left(M_{n}\right)}(\Omega)$, we put $\tilde{D}^{\alpha} f$ for the element of $L^{p}(\Omega)$ to which $D^{\alpha} f$ belongs, $\alpha \in \mathbb{N}_{0}^{k}$. By $V_{r}$ we represent the linear subspace of $Z_{r}$ formed by those families ( $\tilde{D}^{\alpha} f: \alpha \in \mathbb{N}_{0}^{k}$ ) such that $f \in \mathcal{B}_{L^{p}}^{\left(M_{n}\right), 1 / r}(\Omega)$. Let

$$
\Phi_{r}: \mathcal{B}_{L^{p}}^{\left(M_{n}\right), p, 1 / r}(\Omega) \longrightarrow V_{r}
$$

be such that

$$
\Phi_{r}(f)=\left(\tilde{D}^{\alpha} f\right), \quad f \in \mathcal{B}_{L^{p}}^{\left(M_{n}\right), 1 / r}(\Omega) .
$$

Then, $\Phi_{r}$ is a linear onto isometry. We put $V:=\cap\left\{V_{r}: r \in \mathbb{N}\right\}$ considered as a subspace of $Z$. Let

$$
\Phi: \mathcal{B}_{L^{p}}^{\left(M_{n}\right)}(\Omega) \longrightarrow V
$$

be such that

$$
\Phi(f)=\left(\tilde{D}^{\alpha} f\right), \quad f \in \mathcal{B}_{L^{p}}^{\left(M_{n}\right)}(\Omega) .
$$

Clearly, $\Phi$ is a topological isomorphism from $\mathcal{B}_{L^{p}}^{\left(M_{n}\right)}(\Omega)$ onto $V$.

In the following, we fix $1 \leq p<\infty$. By $q$ we denote the conjugate of $p$, i.e., $q=\infty$ when $p=1$, and, if $p>1$ then $\frac{1}{p}+\frac{1}{q}=1$. 
Proposition 3. For each $j$ in a set $J$, let $\left(f_{\alpha, j}: \alpha \in \mathbb{N}_{0}^{k}\right)$ be a family of elements of $\mathcal{L}^{q}(\Omega)$ such that there is $h>0$ with

$$
\sup _{\substack{\alpha \in \mathbb{N}_{j}^{k} \\ j \in J}} h^{|\alpha|} M_{|\alpha|}\left\|f_{\alpha, j}\right\|_{q}<\infty
$$

Then, there is a bounded subset $\left\{S_{j}: j \in J\right\}$ of $\mathcal{B}_{L^{p}}^{\left(M_{n}\right)^{\prime}}(\Omega)$ such that

$$
\left\langle\varphi, S_{j}\right\rangle=\sum_{\alpha \in \mathbb{N}_{0}^{k}} \int_{\Omega} D^{\alpha} \varphi \cdot f_{\alpha, j} \mathrm{~d} x, \quad j \in J, \quad \varphi \in \mathcal{B}_{L^{p}}^{\left(M_{n}\right)}(\Omega),
$$

where the series converges absolutely and uniformly when $j$ varies in $J$ and $\varphi$ varies in any given bounded subset of $\mathcal{B}_{L^{p}}^{\left(M_{n}\right)}(\Omega)$.

Proof. We identify in the usual fashion $f_{\alpha, j}$ with a continuous linear functional on $L^{p}(\Omega)$ whose norm is $\left\|f_{\alpha, j}\right\|_{q}$. We apply Proposition 2 to obtain, for each $j$ in $J$, a unique element $u_{j}$ in $Z^{\prime}$ whose restriction to $Z^{\alpha}$ coincides with $f_{\alpha, j}, \alpha \in \mathbb{N}_{0}^{k}$. If we fix $j$ in $J$, we apply Proposition 1 for $M=\left\{u_{j}\right\}$ and so obtain that

$$
\left\langle\left(\tilde{g}_{\alpha}\right), u_{j}\right\rangle=\sum_{\alpha \in \mathbb{N}_{0}^{k}} \int_{\Omega} g_{\alpha} \cdot f_{\alpha, j} \mathrm{~d} x, \quad g_{\alpha} \in \tilde{g}_{\alpha}, \quad\left(\tilde{g}_{\alpha}\right) \in Z .
$$

We find $r \in \mathbb{N}$ such that $1 / r<h$. We fix $\left(\tilde{g}_{\alpha}\right)$ in $Z$. We then have

$$
\begin{aligned}
\left|\left\langle\left(\tilde{g}_{\alpha}\right), u_{j}\right\rangle\right| & \leq \sum_{\alpha \in \mathbb{N}_{0}^{k}} \int_{\Omega}\left|g_{\alpha}\right| \cdot\left|f_{\alpha, j}\right| \mathrm{d} x \\
& \leq \sum_{\alpha \in \mathbb{N}_{0}^{k}}\left\|g_{\alpha}\right\|_{p} \cdot\left\|f_{\alpha, j}\right\|_{q} \\
& \leq \sum_{\alpha \in \mathbb{N}_{0}^{k}} \frac{(2 k r)^{|\alpha|}\left\|g_{\alpha}\right\|_{p}}{M_{|\alpha|}} \frac{1}{(2 k)^{|\alpha|}} r^{-|\alpha|} M_{|\alpha|}\left\|f_{\alpha, j}\right\|_{q} \\
& \leq \sum_{\alpha \in \mathbb{N}_{0}^{k}}\left\|\left(\tilde{g}_{\alpha}\right)\right\|_{2 k r} \cdot \frac{1}{(2 k)^{|\alpha|}} \sup _{\substack{\gamma \in \mathbb{N}_{0}^{k} \\
j \in J}} h^{|\gamma|} M_{|\gamma|}\left\|f_{\gamma, j}\right\|_{q} \\
& =2\left\|\left(\tilde{g}_{\alpha}\right)\right\|_{2 k r} \cdot \sup _{\substack{\gamma \in \mathbb{N}_{0}^{k} \\
j \in J}} h^{|\gamma|} M_{|\gamma|} \mid\left\|f_{\gamma, j}\right\|_{q}
\end{aligned}
$$

and thus

$$
\sup _{j \in J} \mid\left\langle\left(\tilde{g}_{\alpha}\right), u_{j}\right\rangle<\infty
$$

Applying now the Theorem of Banach-Steinhaus, we obtain that $\left\{u_{j}: j \in J\right\}$ is a bounded subset of $Z^{\prime}$. Proposition 1 yields that, for $M=\left\{u_{j}: j \in J\right\}$, the series in (2) converges absolutely and uniformly when $j$ varies in $J$ and $\left(\tilde{g}_{\alpha}\right)$ varies in any given bounded subset of $Z$.

We put $w$ for the mapping $\Phi$ considered from $\mathcal{B}_{L^{p}}^{\left(M_{n}\right)}(\Omega)$ into $Z$. Let ${ }^{t} w$ be the transpose of $w$. We write

$$
S_{j}:={ }^{t} w\left(u_{j}\right), \quad j \in J .
$$

Then $\left\{S_{j}: j \in J\right\}$ is a bounded subset of $\mathcal{B}_{L^{p}}^{\left(M_{n}\right)^{\prime}}(\Omega)$. On the other hand, for each $\varphi \in \mathcal{B}_{L^{p}}^{\left(M_{n}\right)}(\Omega)$, we have

$$
\left\langle\left(\tilde{D}^{\alpha} \varphi\right), u_{j}\right\rangle=\left\langle w(\varphi), u_{j}\right\rangle=\left\langle\varphi,{ }^{t} w\left(u_{j}\right)\right\rangle=\left\langle\varphi, S_{j}\right\rangle .
$$


Consequently, for each $\varphi$ of $\mathcal{B}_{L^{p}}^{\left(M_{n}\right)}(\Omega)$ and each $j \in J$, it follows

$$
\left\langle\varphi, S_{j}\right\rangle=\left\langle\left(\tilde{D}^{\alpha} \varphi\right), u_{j}\right\rangle=\sum_{\alpha \in \mathbb{N}_{0}^{k}} D^{\alpha} \varphi \cdot f_{\alpha, j} \mathrm{~d} x
$$

Finally, when $\varphi$ varies in a bounded subset of $\mathcal{B}_{L^{p}}^{\left(M_{n}\right)}(\Omega),\left(\tilde{D}^{\alpha} \varphi\right)$ varies in a bounded subset of $Z$. The conclusion is now obvious.

Proposition 4. If $\left\{S_{j}: j \in J\right\}$ is a bounded subset of $\mathcal{B}_{L^{p}}^{\left(M_{n}\right)^{\prime}}(\Omega)$, there are $h>0$ and, for each $j \in J$, a family $\left(f_{\alpha, j}: \alpha \in \mathbb{N}_{0}^{k}\right)$ of elements of $\mathcal{L}^{q}(\Omega)$ such that

$$
\sup _{\substack{\alpha \in \mathbb{N}_{J}^{k} \\ j \in J}} h^{|\alpha|} M_{|\alpha|} \mid\left\|f_{\alpha, j}\right\|_{q}<\infty
$$

and

$$
\left\langle\varphi, S_{j}\right\rangle=\sum_{\alpha \in \mathbb{N}_{0}^{k}} \int_{\Omega} D^{\alpha} \varphi \cdot f_{\alpha, j} \mathrm{~d} x, \quad j \in J, \quad \varphi \in \mathcal{B}_{L^{p}}^{\left(M_{n}\right)}(\Omega),
$$

where the series converges absolutely and uniformly when $j$ varies in $J$ and $\varphi$ varies in any given bounded subset of $\mathcal{B}_{L^{p}}^{\left(M_{n}\right)}(\Omega)$.

PRoOF. We have

$$
{ }^{t} w: Z^{\prime} \longrightarrow \mathcal{B}_{L^{p}}^{\left(M_{n}\right)^{\prime}}(\Omega)
$$

is onto. It is easy to verify that there is a bounded subset $\left\{u_{j}: j \in J\right\}$ in $Z^{\prime}$ such that

$$
{ }^{t} w\left(u_{j}\right)=S_{j}, \quad j \in J .
$$

We put $f_{\alpha, j}$ for the element of $\mathcal{L}^{q}(\Omega)$ given by the restriction of $u_{j}$ to $X^{\alpha}$. We apply Proposition 1 for $M=\left\{u_{j}: j \in J\right\}$ and so obtain $r \in \mathbb{N}$ such that

$$
\sup _{\substack{\alpha \in \mathbb{N}_{0}^{k} \\ j \in J}} r^{-|\alpha|} M_{|\alpha|}\left\|f_{\alpha, j}\right\|_{q}<\infty
$$

and

$$
\left\langle\left(\tilde{D}^{\alpha} \varphi\right), u_{j}\right\rangle=\sum_{\alpha \in \mathbb{N}_{0}^{k}}\left\langle\tilde{D}^{\alpha} \varphi, \tilde{f}_{\alpha, j}\right\rangle=\sum_{\alpha \in \mathbb{N}_{0}^{k}} \int_{\Omega} D^{\alpha} \varphi \cdot f_{\alpha, j} \mathrm{~d} x, \quad j \in J, \quad \varphi \in \mathcal{B}_{L^{p}}^{\left(M_{n}\right)}(\Omega),
$$

where the series converges absolutely and uniformly when $j$ varies in $J$ and $\varphi$ varies in any given bounded subset of $\mathcal{B}_{L^{p}}^{\left(M_{n}\right)}(\Omega)$. Finally, for each $j \in J$, we have

$$
\left\langle\left(\tilde{D}^{\alpha} \varphi\right), u_{j}\right\rangle=\left\langle w(\varphi), u_{j}\right\rangle=\left\langle\varphi,{ }^{t} w\left(u_{j}\right)\right\rangle=\left\langle\varphi, S_{j}\right\rangle
$$

and the conclusion follows.

Given a compact subset $K$ of $\Omega$ and $r \in \mathbb{N}$, we put $\mathcal{D}_{\left(L^{p}\right)}^{\left(M_{n}\right), 1 / r}(K)$ to denote the subspace of $\mathcal{B}_{L^{p}}^{\left(M_{n}\right), 1 / r}(\Omega)$ whose elements have their support in $K$. If $\left(f_{m}\right)$ is a sequence in $\mathcal{D}_{(L)}^{\left(M_{n}\right), 1 / r}(K)$ which converges to $f$ in $\mathcal{B}_{L^{p}}^{\left(M_{n}\right), 1 / r}(\Omega)$, there is a subsequence $\left(f_{m_{i}}\right)$ of $\left(f_{m}\right)$ which converges to $f$ almost everywhere. Since $f_{m_{i}}(x)=0, x \in \Omega \backslash K$, we have that $f$ belongs to $\mathcal{D}_{\left(L^{p}\right)}^{\left(M_{n}\right), 1 / r}(K)$, from where we get that this space is a Banach space. We put $\mathcal{D}_{\left(L^{p}\right)}^{\left(M_{n}\right)}(K)$ for the projective limit of the sequence $\left(\mathcal{D}_{\left(L^{p}\right)}^{\left(M_{n}\right), 1 / r}(K)\right)$ of Banach 
spaces. It is immediate that $\mathcal{D}_{\left(L^{p}\right)}^{\left(M_{n}\right)}(K)$ coincides with the subspace of $\mathcal{B}_{L^{p}}^{\left(M_{n}\right)}(\Omega)$ formed by the functions with support in $K$. We now write

$$
\mathcal{D}_{\left(L^{p}\right)}^{\left(M_{n}\right)}(\Omega):=\bigcup_{r=1}^{\infty} \mathcal{D}_{\left(L^{p}\right)}^{\left(M_{n}\right)}\left(K_{r}\right)
$$

and assume that $\mathcal{D}_{\left(L^{p}\right)}^{\left(M_{n}\right)}(\Omega)$ is the inductive limit of the sequence $\left(\mathcal{D}_{\left(L^{p}\right)}^{\left(M_{n}\right)}\left(K_{r}\right)\right)$ of Fréchet spaces. We also assume that the topological dual $\mathcal{D}_{\left(L^{p}\right)}^{\left(M_{n}\right)^{\prime}}(\Omega)$ of $\mathcal{D}_{\left(L^{p}\right)}^{\left(M_{n}\right)}(\Omega)$ is endowed with the strong topology.

If $g \in \mathcal{L}^{p_{1}}\left(\mathbb{R}^{k}\right)$ and $l \in \mathcal{L}^{p_{2}}\left(\mathbb{R}^{k}\right)$, with $1 \leq p_{1}, p_{2} \leq \infty$ and $1 / p_{1}+1 / p_{2} \geq 1$, then the convolution of $g$ and $l$ exists almost everywhere. We extend this convolution to the whole of $\mathbb{R}^{k}$ by assigning the zero value for the points where it is not defined. Thus $g * l$ belongs to $\mathcal{L}^{s}\left(\mathbb{R}^{k}\right)$, where $\frac{1}{s}=\frac{1}{p_{1}}+\frac{1}{p_{2}}-1$ and we then have

$$
\|g * l\|_{s} \leq\|g\|_{p_{1}} \cdot\|l\|_{p_{2}} .
$$

This property will be used in the proof of the next result.

Proposition 5. The linear space $\mathcal{D}^{\left(M_{n}\right)}(\Omega)$ is dense in $\mathcal{D}_{\left(L^{p}\right)}^{\left(M_{n}\right)}(\Omega)$.

ProOF. We may assume that $\stackrel{\circ}{K}_{1} \neq \emptyset$ and that $K_{m} \subset \stackrel{\circ}{K}_{m+1}, m=1,2, \ldots$ Given $\rho>0$, we write $B(\rho)$ for the closed ball in $\mathbb{R}^{k}$ with center in the origin and radius $\rho$. We take $f$ in $\mathcal{D}_{\left(L^{p}\right)}^{\left(M_{n}\right)}(\Omega)$. We find a positive integer $m$ such that $f \in \mathcal{D}_{\left(L^{p}\right)}^{\left(M_{n}\right)}\left(K_{m}\right)$. We choose a sequence $\left(\psi_{i}\right)$ in $\mathcal{D}^{\left(M_{n}\right)}\left(\mathbb{R}^{k}\right)$ satisfying:

(i) $\psi_{i}(x) \geq 0, x \in \mathbb{R}^{k}$.

(ii) $\int_{\mathbb{R}^{k}} \psi_{i}(x) \mathrm{d} x=1$.

(iii) $\operatorname{supp} \psi_{i} \subset B\left(\rho_{i}\right), \rho_{1}>\rho_{2}>\cdots>\rho_{i}>\cdots$,

$$
\lim _{i} \rho_{i}=0
$$

and $K_{m}+B\left(\rho_{1}\right) \subset K_{m+1}$.

We extend $f$ to $\mathbb{R}^{k}$ by putting $f(x)=0, x \in \mathbb{R}^{k} \backslash \Omega$. We set $f_{i}:=f * \psi_{i}, i \in \mathbb{N}$. We see next that $\left(f_{i}\right)$ is a sequence in $\mathcal{D}^{\left(M_{n}\right)}\left(K_{m+1}\right)$ which converges to $f$ in $\mathcal{D}_{\left(L^{p}\right)}^{\left(M_{n}\right)}\left(K_{m+1}\right)$. For each $\alpha \in \mathbb{N}_{0}^{k}$, we have

$$
D^{\alpha} f_{i}(x)=\int_{\mathbb{R}^{k}} f(y)\left(D^{\alpha} \psi_{i}\right)(x-y) \mathrm{d} y, \quad x \in \mathbb{R}^{k},
$$

and hence $f_{i}$ is in $\mathcal{D}^{\left(M_{n}\right)}\left(K_{m+1}\right)$.

Let us take $\varepsilon>0$ and $r \in \mathbb{N}$. We find a positive integer $s_{0}$ such that

$$
\left(\frac{r}{r+1}\right)^{s_{0}}\|f\|_{p, 1 / r+1}<\frac{\varepsilon}{4}
$$

Given $\alpha \in \mathbb{N}_{0}^{k}$, we have, for $x \in \mathbb{R}^{k}$,

$$
\begin{aligned}
\left|D^{\alpha} f_{i}(x)-D^{\alpha} f(x)\right| & \leq \int_{\mathbb{R}^{k}}\left|\left(D^{\alpha} f\right)(x-y)-D^{\alpha}(x)\right| \cdot \psi_{i}(y) \mathrm{d} y \\
& \leq \sup \left\{\left|\left(D^{\alpha} f\right)(x-y)-D^{\alpha} f(x)\right|: y \in B\left(\delta_{i}\right)\right\}
\end{aligned}
$$

and so, if $\mu$ is the Lebesgue measure in $\mathbb{R}^{k}$, we may find $i_{0} \in \mathbb{N}$ such that

$$
\left|D^{\alpha} f_{i}(x)-D^{\alpha} f(x)\right|<\frac{\varepsilon}{2 r^{s_{0}} \mu\left(K_{m+1}\right)}, \quad i \geq i_{0}, \quad x \in \mathbb{R}^{k}, \quad|\alpha| \leq s_{0} .
$$


Then

$$
\left\|D^{\alpha} f_{i}-D^{\alpha} f\right\|_{p} \leq \frac{\varepsilon}{2 r^{s_{0}}}, \quad i \geq i_{0}, \quad|\alpha| \leq s_{0} .
$$

Applying (3) for $p_{1}=p, p_{2}=1, g=D^{\alpha} f$ and $l=\psi_{i}$, we obtain

$$
\left\|D^{\alpha} f_{i}\right\|_{p}=\left\|\left(D^{\alpha} f\right) * \psi_{i}\right\|_{p} \leq\left\|D^{\alpha} f\right\|_{p} \cdot\left\|\psi_{i}\right\|_{1}=\left\|D^{\alpha} f\right\|_{p} .
$$

Therefore, for $i \geq i_{0}$, we have that

$$
\begin{aligned}
\left\|f-f_{i}\right\|_{p, 1 / r} & =\sup _{\alpha \in \mathbb{N}_{0}^{k}} \frac{r^{|\alpha|}\left\|D^{\alpha}\left(f-f_{i}\right)\right\|_{p}}{M_{|\alpha|}} \\
& \leq \sup _{|\alpha| \leq s_{0}} \frac{r^{|\alpha|}\left\|D^{\alpha}\left(f-f_{i}\right)\right\|_{p}}{M_{|\alpha|}}+\sup _{|\alpha|>s_{0}} \frac{r^{|\alpha|}\left\|D^{\alpha}\left(f-f_{i}\right)\right\|_{p}}{M_{|\alpha|}} \\
& \leq \frac{\varepsilon}{2}+\sup _{|\alpha|>s_{0}}\left(\frac{r}{r+1}\right)^{|\alpha|} \frac{(r+1)^{|\alpha|}\left(\left\|D^{\alpha} f\right\|_{p}+\left\|D^{\alpha} f_{i}\right\|_{p}\right)}{M_{|\alpha|}} \\
& \leq \frac{\varepsilon}{2}+\left(\frac{r}{r+1}\right)^{s_{0}} \sup _{\alpha \in \mathbb{N}_{0}^{k}} \frac{2(r+1)^{|\alpha|} \mid D^{\alpha} f \|_{p}}{M_{|\alpha|}} \\
& =\frac{\varepsilon}{2}+\left(\frac{r}{r+1}\right)^{s_{0}} \cdot 2\|f\|_{p, 1 / r+1} \\
& <\varepsilon
\end{aligned}
$$

from where the conclusion follows. tions.

The previous proposition tells us that the elements of $\mathcal{D}_{\left(L^{p}\right)}^{\left(M_{n}\right)^{\prime}}(\Omega)$ may be considered as ultradistribuProposition 6. If $\varphi \in \mathcal{B}_{\left(L^{p}\right)}^{\left(M_{n}\right)}(\Omega)$ and $g \in \mathcal{D}^{\left(M_{n}\right)}(\Omega)$, then $g \varphi$ is in $\mathcal{D}_{\left(L^{p}\right)}^{\left(M_{n}\right)}(\Omega)$.

ProOF. The support of $g \varphi$ is a compact subset of $\Omega$. We take a positive integer $r$. We find a constant $C_{r}$ such that

$$
\left|D^{\alpha} g(x)\right| \leq C_{r}(2 r)^{-|\alpha|} M_{|\alpha|}, \quad x \in \Omega, \quad \alpha \in \mathbb{N}_{0}^{k}
$$

We have that, for each $\alpha \in \mathbb{N}_{0}^{k}$,

$$
\begin{aligned}
\left\|D^{\alpha}(g \varphi)\right\|_{p} & \leq \sum_{\beta \leq \alpha} \frac{\alpha !}{\beta !(\alpha-\beta) !}\left\|D^{\beta} g \cdot D^{\alpha-\beta} \varphi\right\|_{p} \\
& \leq \sum_{\beta \leq \alpha} \frac{\alpha !}{\beta !(\alpha-\beta) !} C_{r}(2 r)^{-|\beta|} M_{|\beta|}\left\|D^{\alpha-\beta} \varphi\right\|_{p} \\
& \leq C_{r} \sum_{\beta \leq \alpha} \frac{\alpha !}{\beta !(\alpha-\beta) !}(2 r)^{-|\beta|} M_{|\beta|}\|\varphi\|_{p, 1 / 2 r} M_{|\alpha-\beta|}(2 r)^{-|\alpha-\beta|} \\
& \leq C_{r} \sum_{\beta \leq \alpha} \frac{\alpha !}{\beta !(\alpha-\beta) !}(2 r)^{-|\alpha|} M_{|\alpha|}\|\varphi\|_{p, 1 / 2 r}=C_{r}\|\varphi\|_{p, 1 / 2 r} r^{-|\alpha|} M_{|\alpha|}
\end{aligned}
$$

and thus

$$
\sup _{\alpha \in \mathbb{N}_{0}^{k}} \frac{r^{|\alpha|}\left\|D^{\alpha}(g \varphi)\right\|_{p}}{M_{|\alpha|}} \leq C_{r}\|\varphi\|_{p, 1 / 2 r}
$$

and the conclusion follows. 
In what follows, before stating our next lemma, we shall give the details of a previous construction. We take a bounded subset $\left\{S_{j}: j \in J\right\}$ of $\mathcal{D}_{\left(L^{p}\right)}^{\left(M_{n}\right)^{\prime}}(\Omega)$ so that there is a compact subset $H$ of $\Omega$ with

$$
\operatorname{supp} S_{j} \subset H, \quad j \in J
$$

Let $K$ be a compact subset of $\Omega$ with $H \subset \stackrel{\circ}{K}$. We choose an element $\eta$ of $\mathcal{D}^{\left(M_{n}\right)^{\prime}}(\Omega)$ which takes value one in a neighborhood of $K$ and whose support is compact. For each $\varphi \in \mathcal{B}_{L^{p}}^{\left(M_{n}\right)}(\Omega)$, we have that, after the previous proposition, $\eta \varphi$ is in $\mathcal{D}_{\left(L^{p}\right)}^{\left(M_{n}\right)}(\Omega)$. We put

$$
\left\langle\varphi, W_{j}\right\rangle:=\left\langle\eta \varphi, S_{j}\right\rangle, \quad j \in J, \quad \varphi \in \mathcal{B}_{\left(L^{p}\right)}^{\left(M_{n}\right)}(\Omega)
$$

It is easy to see that $\left\{W_{j}: j \in J\right\}$ is a bounded subset of $\mathcal{B}_{L^{p}}^{\left(M_{n}\right)^{\prime}}(\Omega)$. We apply Proposition 4 to obtain $h>0$ and, for each $j \in J$, a family $\left(f_{\alpha, j}: \alpha \in \mathbb{N}_{0}^{k}\right)$ of elements of $\mathcal{L}^{q}(\Omega)$ such that

$$
\sup _{\substack{\alpha \in \mathbb{N}_{0}^{k} \\ j \in J}} h^{|\alpha|} M_{|\alpha|}\left\|f_{\alpha, j}\right\|_{q}<\infty
$$

and

$$
\left\langle\varphi, W_{j}\right\rangle=\sum_{\alpha \in \mathbb{N}_{0}^{k}} \int_{\Omega} D^{\alpha} \varphi \cdot f_{\alpha, j} \mathrm{~d} x, \quad j \in J, \quad \varphi \in \mathcal{B}_{L^{p}}^{\left(M_{n}\right)}(\Omega) .
$$

Let $g$ be an element of $\mathcal{D}^{\left(M_{n}\right)}(\Omega)$ which takes value one in a neighborhood of $H$ and whose support is contained in $\stackrel{\circ}{K}$. Then, on account of the previous proposition, we have

$$
\begin{aligned}
\left\langle g \varphi, S_{j}\right\rangle & =\left\langle\eta g \varphi, S_{j}\right\rangle=\left\langle g \varphi, W_{j}\right\rangle=\sum_{\alpha \in \mathbb{N}_{0}^{k}} \int_{\Omega} D^{\alpha}(g \varphi) \cdot f_{\alpha, j} \mathrm{~d} x \\
& =\sum_{\alpha \in \mathbb{N}_{0}^{k}} \int_{\Omega}\left(\sum_{\beta \leq \alpha} \frac{\alpha !}{\beta !(\alpha-\beta) !} D^{\beta} g \cdot D^{\alpha-\beta} \varphi\right) \cdot f_{\alpha, j} \mathrm{~d} x, \quad j \in J, \quad \varphi \in \mathcal{B}_{L^{p}}^{\left(M_{n}\right)}(\Omega) .
\end{aligned}
$$

We now take a positive integer $r>\frac{4 k}{h}$. Let $C_{r}$ be a positive constant such that

$$
\left|D^{\beta} g(x)\right| \leq C_{r} r^{-|\beta|} M_{|\beta|}, \quad x \in \Omega, \quad \beta \in \mathbb{N}_{0}^{k} .
$$

We then have that

$$
\begin{aligned}
\sum_{\beta \leq \alpha} \frac{\alpha !}{\beta !(\alpha-\beta) !} & \int_{\Omega}\left|D^{\beta} g\right| \cdot\left|D^{\alpha-\beta} \varphi\right| \cdot\left|f_{\alpha, j}\right| \mathrm{d} x \\
& \leq \sum_{\beta \leq \alpha} \frac{\alpha !}{\beta !(\alpha-\beta) !} C_{r} r^{-|\beta|} M_{|\beta|} \int_{\Omega}\left|D^{\alpha-\beta} \varphi\right| \cdot\left|f_{\alpha, j}\right| \mathrm{d} x \\
& \leq \sum_{\beta \leq \alpha} \frac{\alpha !}{\beta !(\alpha-\beta) !} C_{r} r^{-|\beta|} M_{|\beta|}\left\|D^{\alpha-\beta} \varphi\right\|_{p} \cdot\left\|f_{\alpha, j}\right\|_{q} \\
& \leq \sum_{\beta \leq \alpha} \frac{\alpha !}{\beta !(\alpha-\beta) !} C_{r} r^{-|\beta|} M_{|\beta|}\|\varphi\|_{p, 1 / r} r^{-|\alpha-\beta|} M_{|\alpha-\beta|}\left\|f_{\alpha, j}\right\|_{q} \\
& \leq \sum_{\beta \leq \alpha} \frac{\alpha !}{\beta !(\alpha-\beta) !} C_{r} r^{-|\alpha|} M_{|\alpha|}\|\varphi\|_{p, 1 / r}\left\|f_{\alpha, j}\right\|_{q} \\
& =C_{r}\|\varphi\|_{p, 1 / r}\left(\frac{r}{2}\right)^{-|\alpha|} M_{|\alpha|}\left\|f_{\alpha, j}\right\|_{q}
\end{aligned}
$$




$$
\begin{aligned}
& \leq C_{r}\|\varphi\|_{p, 1 / r} \frac{1}{(2 k)^{|\alpha|}} h^{|\alpha|} M_{|\alpha|}\left\|f_{\alpha, j}\right\|_{q} \\
& \leq C_{r}\|\varphi\|_{p, 1 / r} \frac{1}{(2 k)^{|\alpha|}} \sup _{\substack{\delta \in \mathbb{N}_{0}^{k} \\
j \in J}} h^{|\delta|} M_{|\delta|}\left\|f_{\alpha, j}\right\|_{q}
\end{aligned}
$$

and, noticing that

$$
\sum_{\alpha \in \mathbb{N}_{0}^{k}} \frac{1}{(2 k)^{|\alpha|}}=2
$$

we have that the series (4) converges absolutely and so we may write, putting $\gamma:=\alpha-\beta$,

$$
\begin{aligned}
\sum_{\alpha \in \mathbb{N}_{0}^{k}} \sum_{\beta \leq \alpha} & \frac{\alpha !}{\beta !(\alpha-\beta) !} \int_{\Omega} D^{\beta} g \cdot D^{\alpha-\beta} \varphi \cdot f_{\alpha, j} \mathrm{~d} x \\
& =\sum_{\gamma \in \mathbb{N}_{0}^{k}} \sum_{\beta \in \mathbb{N}_{0}^{k}} \frac{(\beta+\gamma) !}{\beta ! \gamma !} \int_{\Omega} D^{\beta} g \cdot D^{\gamma} \varphi \cdot f_{\beta+\gamma, j} \mathrm{~d} x .
\end{aligned}
$$

Lemma 1. Let $\left\{S_{j}: j \in J\right\}$ be a bounded subset of $\mathcal{D}_{\left(L^{p}\right)}^{\left(M_{n}\right)^{\prime}}(\Omega)$ such that there is a compact subset $H$ in $\Omega$ with

$$
\operatorname{supp} S_{j} \subset H, \quad j \in J .
$$

Let $K$ be a compact subset of $\Omega$ such that $H \subset \stackrel{\circ}{K}$. Then there are $h>0$ and, for each $j \in J$, a family $\left(g_{\alpha, j}: \alpha \in \mathbb{N}_{0}^{k}\right)$ of elements of $\mathcal{L}^{q}(\Omega)$ such that

$$
\begin{gathered}
\sup _{\substack{\alpha \in \mathbb{N}_{0}^{k} \\
j \in J}} h^{|\alpha|} M_{|\alpha|}\left\|g_{\alpha, j}\right\|_{q}<\infty \\
\operatorname{supp} g_{\alpha, j} \subset \stackrel{\circ}{K}, \quad j \in J, \quad \alpha \in \mathbb{N}_{0}^{k},
\end{gathered}
$$

and

$$
\left\langle\varphi, S_{j}\right\rangle=\sum_{\alpha \in \mathbb{N}_{0}^{k}} \int_{\Omega} D^{\alpha} \varphi \cdot g_{\alpha, j} \mathrm{~d} x, \quad j \in J, \quad \varphi \in \mathcal{D}_{\left(L^{p}\right)}^{\left(M_{n}\right)}(\Omega),
$$

where the series converges absolutely and uniformly when $j$ varies in $J$ and $\varphi$ varies in any given bounded subset of $\mathcal{D}_{\left(L^{p}\right)}^{\left(M_{n}\right)}(\Omega)$.

Proof. We choose $h>0$ and, for each $j \in J$, the family $\left(f_{\alpha, j}: \alpha \in \mathbb{N}_{0}^{k}\right)$ of elements of $\mathcal{L}^{q}(\Omega)$ with the properties above cited. We fix $\gamma \in \mathbb{N}_{0}^{k}$ and take $\rho \in \tilde{\rho} \in L^{p}(\Omega)$. We choose $r \in \mathbb{N}, r>4 k / h$. Then

$$
\begin{aligned}
\mid \sum_{\beta \in \mathbb{N}_{0}^{k}} \frac{(\beta+\gamma) !}{\beta ! \gamma !} & \int_{\Omega} D^{\beta} g \cdot \rho \cdot f_{\beta+\gamma, j} \mathrm{~d} x \mid \\
& \leq \sum_{\beta \in \mathbb{N}_{0}^{k}} \frac{(\beta+\gamma) !}{\beta ! \gamma !} \int_{\Omega}\left|D^{\beta} g\right| \cdot|\rho| \cdot\left|f_{\beta+\gamma, j}\right| \mathrm{d} x \\
& \leq \mid \sum_{\beta \in \mathbb{N}_{0}^{k}} \frac{(\beta+\gamma) !}{\beta ! \gamma !} C_{r} r^{-|\beta|} M_{|\beta|}\|\rho\|_{p} \cdot\left\|f_{\beta+\gamma, j}\right\|_{q} \\
& \leq \frac{C_{r}\|\rho\|_{p}}{M_{|\gamma|}} \sum_{\beta \in \mathbb{N}_{0}^{k}} 2^{|\beta+\gamma|} r^{-|\beta|} M_{|\beta+\gamma|}\left\|f_{\beta+\gamma, j}\right\|_{q}
\end{aligned}
$$




$$
\begin{aligned}
& \leq \frac{C_{r}\|\rho\|_{p} r|\gamma|}{M_{|\gamma|}} \sum_{\beta \in \mathbb{N}_{0}^{k}}(r / 2)^{-|\beta+\gamma|} M_{|\beta+\gamma|}\left\|f_{\beta+\gamma, j}\right\|_{q} \\
& \leq \frac{C_{r}\|\rho\|_{p}}{M_{|\gamma|}} r|\gamma| \sum_{\beta \in \mathbb{N}_{0}^{k}}(2 k / h)^{-|\beta+\gamma|} M_{|\beta+\gamma|}\left\|f_{\beta+\gamma, j}\right\|_{q} \\
& \leq \frac{C_{r}\|\rho\|_{p}}{M_{|\gamma|}} r|\gamma|\left(\sup _{\substack{\alpha \in \mathbb{N}_{0}^{k} \\
j \in J}} h^{|\alpha|} M_{|\alpha|}\left\|f_{\alpha, j}\right\|_{q}\right) \sum_{\beta \in \mathbb{N}_{0}^{k}} \frac{1}{(2 k)^{|\beta|}}
\end{aligned}
$$

from where we get that there is $A_{\gamma}>0$ such that

$$
\left|\sum_{\beta \in \mathbb{N}_{0}^{k}} \frac{(\beta+\gamma) !}{\beta ! \gamma !} \int_{\Omega} D^{\beta} g \cdot \rho \cdot f_{\beta+\gamma, j} \mathrm{~d} x\right| \leq A_{\gamma}\|\tilde{\rho}\|_{p} .
$$

If we put, for each $\rho \in \tilde{\rho} \in L^{p}(\Omega)$,

$$
v_{\gamma, j}(\tilde{\rho}):=\sum_{\beta \in \mathbb{N}_{0}^{k}} \frac{(\beta+\gamma) !}{\beta ! \gamma !} \int_{\Omega} D^{\beta} g \cdot \rho \cdot f_{\beta+\gamma, j} \mathrm{~d} x,
$$

we then have that $v_{\gamma, j}$ is a complex function defined in $L^{p}(\Omega)$, clearly linear, such that after (6) is also continuous. Then there is $g_{\gamma, j}$ in $\mathcal{L}^{q}(\Omega)$ such that

$$
v_{\gamma, j}(\tilde{\rho})=\int_{\Omega} \rho \cdot g_{\gamma, j} \mathrm{~d} x, \quad \rho \in \tilde{\rho} \in L^{p}(\Omega) .
$$

If $M$ is the support of $g$, then it is clear that

$$
\operatorname{supp} g_{\alpha, j} \subset M \subset \stackrel{\circ}{K}, \quad j \in J, \quad \gamma \in \mathbb{N}_{0}^{k} .
$$

For each $\varphi \in \mathcal{B}_{L^{p}}^{\left(M_{n}\right.}(\Omega)$, we have

$$
\sum_{\beta \in \mathbb{N}_{0}^{k}} \frac{(\beta+\gamma) !}{\beta ! \gamma !} \int_{\Omega} D^{\beta} g \cdot D^{\gamma} \varphi \cdot f_{\beta+\gamma, j} \mathrm{~d} x=\int_{\Omega} D^{\gamma} \varphi \cdot g_{\gamma, j} \mathrm{~d} x
$$

and, by (4) and (5),

$$
\left\langle g \varphi, S_{j}\right\rangle=\sum_{\gamma \in \mathbb{N}_{0}^{k}} \int_{\Omega} D^{\gamma} \varphi \cdot g_{\alpha, j} \mathrm{~d} x, \quad j \in J, \quad \varphi \in \mathcal{B}_{L^{p}}^{\left(M_{n}\right)}(\Omega) .
$$

We now fix $\gamma \in \mathbb{N}_{0}^{k}$ and $j \in J$. We choose $\tilde{\rho} \in L^{p}(\Omega)$ such that $\|\tilde{\rho}\|_{p}<2$ and $v_{\gamma, j}(\tilde{\rho})=\left\|g_{\gamma, j}\right\|_{q}$. We take $r \in \mathbb{N}$ with $r>4 k / h$. If we put

$$
C:=2 \sup _{\alpha \in \mathbb{N}_{0}^{k}} h^{|\alpha|} M_{|\alpha|}\left\|f_{\alpha, j}\right\|_{q}
$$

we have obtained above

$$
\left|\sum_{\beta \in \mathbb{N}_{0}^{k}} \frac{(\beta+\gamma) !}{\beta ! \gamma !} \int_{\Omega} D^{\beta} g \cdot \rho \cdot f_{\beta+\gamma, j} \mathrm{~d} x\right| \leq \frac{C_{r}\|\tilde{\rho}\|_{p} r|\gamma|}{M_{|\gamma|}} \cdot C .
$$


Consequently,

$$
\begin{aligned}
r^{-|\gamma|} M_{|\gamma|}\left\|g_{\alpha, j}\right\|_{q} & =r^{-|\gamma|} M_{|\gamma|} v_{\gamma, j}(\tilde{\rho}) \\
& =r^{-|\gamma|} M_{|\gamma|}\left|\sum_{\beta \in \mathbb{N}_{0}^{k}} \frac{(\beta+\gamma) !}{\beta ! \gamma !} \int_{\Omega} D^{\beta} g \cdot \rho \cdot f_{\beta+\gamma, j} \mathrm{~d} x\right| \\
& \leq r^{-|\gamma|} M_{|\gamma|} \frac{C_{r}\|\rho\|_{p} r|\gamma|}{M_{|\gamma|}} \\
& =2 C_{r} C
\end{aligned}
$$

and so

$$
\sup _{\substack{\gamma \in \mathbb{N}_{0}^{k} \\ j \in J}} r^{-|\gamma|} M_{|\gamma|}|| g_{\alpha, j} \|_{q} \leq 2 C_{r} C .
$$

We apply now Proposition 3 to the families $\left(g_{\alpha, j}: \alpha \in \mathbb{N}_{0}^{k}\right), j \in J$, and so obtain, for each $j \in J$, an element $T_{j}$ in $\mathcal{B}_{L^{p}}^{\left(M_{n}\right)^{\prime}}(\Omega)$ such that

$$
\left\langle\varphi, T_{j}\right\rangle=\sum_{\alpha \in \mathbb{N}_{0}^{K}} \int_{\Omega} D^{\alpha} \varphi \cdot g_{\alpha, j} \mathrm{~d} x, \quad j \in J, \quad \varphi \in \mathcal{B}_{L^{p}}^{\left(M_{n}\right)}(\Omega),
$$

where the series converges absolutely and uniformly when $j$ varies in $J$ and $\varphi$ varies in any given bounded subset of $\mathcal{B}_{L^{p}}^{\left(M_{n}\right)}(\Omega)$. On the other hand, we have

$$
\left\langle\varphi, S_{j}\right\rangle=\left\langle g \varphi, S_{j}\right\rangle=\sum_{\alpha \in \mathbb{N}_{0}^{K}} \int_{\Omega} D^{\alpha} \varphi \cdot g_{\alpha, j} \mathrm{~d} x=\left\langle\varphi, T_{j}\right\rangle
$$

The conclusion is now obvious.

We now put $\mathcal{L}_{\text {loc }}^{q}(\Omega)$ for the linear space over $\mathbb{C}$ of the complex functions defined in $\Omega$ such that, for each compact subset $K$ of $\Omega, f_{\mid K}$ belongs to $\mathcal{L}^{q}(K)$. We write $|f|_{K, q}:=\left\|f_{\mid K}\right\|_{q}$.

Theorem 1. For each $j$ in a set $J$, let $\left(f_{\alpha, j}: \alpha \in \mathbb{N}_{0}^{k}\right)$ be a family of elements of $\mathcal{L}_{\mathrm{loc}}^{q}(\Omega)$ such that, given any compact subset $K$ of $\Omega$, there is $h>0$ such that

$$
\sup _{\substack{\alpha \in \mathbb{N}_{0}^{k} \\ j \in J}} h^{|\alpha|} M_{|\alpha|}\left|f_{\alpha, j}\right|_{K, q}<\infty .
$$

Then, there is a bounded subset $\left\{S_{j}: j \in J\right\}$ in $\mathcal{D}_{\left(L^{p}\right)}^{\left(M_{n}\right)^{\prime}}(\Omega)$ such that

$$
\left\langle\varphi, S_{j}\right\rangle=\sum_{\alpha \in \mathbb{N}_{0}^{K}} \int_{\Omega} D^{\alpha} \varphi \cdot f_{\alpha, j} \mathrm{~d} x, \quad j \in J, \quad \varphi \in \mathcal{D}_{\left(L^{p}\right)}^{\left(M_{n}\right)}(\Omega),
$$

where the series converges absolutely and uniformly when $j$ varies in $J$ and $\varphi$ varies in any given bounded subset of $\mathcal{D}_{\left(L^{p}\right)}^{\left(M_{n}\right)}(\Omega)$.

Proof. For each $m \in \mathbb{N}$, we put

$$
f_{\alpha, j}^{m}:=f_{\alpha, j \mid K_{m}}, \quad \alpha \in \mathbb{N}_{0}^{k}, \quad j \in J .
$$


We apply Proposition 3 and thus obtain a bounded subset $\left\{S_{j}^{m}: j \in J\right\}$ of $\mathcal{B}_{L^{p}}^{\left(M_{n}\right)^{\prime}}\left(\stackrel{\circ}{K_{m}}\right)$ such that

$$
\left\langle\varphi, S_{j}^{m}\right\rangle=\sum_{\alpha \in \mathbb{N}_{0}^{K}} \int_{\Omega} D^{\alpha} \varphi \cdot f_{\alpha, j}^{m} \mathrm{~d} x, \quad j \in J, \quad \varphi \in \mathcal{B}_{L^{p}}^{\left(M_{n}\right)}\left(\stackrel{\circ}{K_{m}}\right),
$$

where the series converges absolutely and uniformly when $j$ varies in $J$ and $\varphi$ varies in any given bounded subset of $\mathcal{D}_{\left(L^{p}\right)}^{\left(M_{n}\right)}\left(\stackrel{\circ}{K_{m}}\right)$.

For a given element $\varphi$ of $\mathcal{D}_{\left(L^{p}\right)}^{\left(M_{n}\right)}(\Omega)$, we find $m \in \mathbb{N}$ such that

$$
\operatorname{supp} \varphi \subset \stackrel{\circ}{K}_{m}
$$

and set

$$
\left\langle\varphi, S_{j}\right\rangle:=\left\langle\varphi, S_{j}^{m}\right\rangle .
$$

It is easy to see that $S_{j}$ is well defined and that $\left\{S_{j}: J \in J\right\}$ is a bounded subset of $\mathcal{D}_{\left(L^{p}\right)}^{\left(M_{n}\right)^{\prime}}(\Omega)$, which leads us to the desired result.

Theorem 2. If $\left\{S_{j}: j \in J\right\}$ is a bounded subset of $\mathcal{D}_{\left(L^{p}\right)}^{\left(M_{n}\right)^{\prime}}(\Omega)$, then there is, for each $j \in J$, a family $\left(f_{\alpha, j}: \alpha \in \mathbb{N}_{0}^{k}\right)$ in $\mathcal{L}_{\text {loc }}^{p}(\Omega)$ such that, given any compact subset $K$ of $\Omega$, there is $h>0$ with

$$
\sup _{\substack{\alpha \in \mathbb{N}_{0}^{k} \\ j \in J}} h^{|\alpha|} M_{|\alpha|}\left|f_{\alpha, j}\right|_{K, q}<\infty
$$

and

$$
\left\langle\varphi, S_{j}\right\rangle=\sum_{\alpha \in \mathbb{N}_{0}^{K}} \int_{\Omega} D^{\alpha} \varphi \cdot f_{\alpha, j}^{m} \mathrm{~d} x, \quad j \in J, \quad \varphi \in \mathcal{D}_{\left(L^{p}\right)}^{\left(M_{n}\right)}(\Omega),
$$

where the series converges absolutely and uniformly when $j$ varies in $J$ and $\varphi$ varies in any given bounded subset of $\mathcal{D}_{\left(L^{p}\right)}^{\left(M_{n}\right)}(\Omega)$.

PRoOF. Let $\left\{O_{m}: m \in \mathbb{N}\right\}$ be a locally finite open covering of $\Omega$ such that $O_{m}$ is relatively compact in $\Omega, m \in \mathbb{N}$. Let $\left\{g_{m}: m \in \mathbb{N}\right\}$ be a partition of unity of class $\left(M_{n}\right)$ subordinated to that covering. It follows that $\left\{g_{m} S_{j}: j \in J\right\}$ is a bounded subset of $\mathcal{D}_{\left(L^{p}\right)}^{\left(M_{n}\right)^{\prime}}(\Omega)$ whose elements have their supports contained in a compact subset of $O_{m}$. Applying the previous lemma, we obtain, for each $j \in J$, a family $\left(f_{\alpha, j}^{m}: \alpha \in \mathbb{N}_{0}^{k}\right)$ of elements of $\mathcal{L}^{q}(\Omega)$ such that there is $h_{m}>0$ with

$$
\begin{gathered}
\sup _{\substack{\alpha \in \mathbb{N}_{0}^{k} \\
j \in J}} h_{m}^{|\alpha|} M_{|\alpha|}\left\|f_{\alpha, j}^{m}\right\|_{q}<\infty, \\
\operatorname{supp} f_{\alpha, j}^{m} \subset O_{m}, \quad j \in J, \quad \alpha \in \mathbb{N}_{0}^{k},
\end{gathered}
$$

and

$$
\left\langle\varphi, g_{m} S_{j}\right\rangle=\sum_{\alpha \in \mathbb{N}_{0}^{K}} \int_{\Omega} D^{\alpha} \varphi \cdot f_{\alpha, j}^{m} \mathrm{~d} x, \quad j \in J, \quad \varphi \in \mathcal{D}_{\left(L^{p}\right)}^{\left(M_{n}\right)}(\Omega),
$$

where the series converges absolutely and uniformly when $j$ varies in $J$ and $\varphi$ varies in any given bounded subset of $\mathcal{D}_{\left(L^{p}\right)}^{\left(M_{n}\right)}(\Omega)$. We put, for each $x \in \Omega, \alpha \in \mathbb{N}_{0}^{k}, j \in J$,

$$
f_{\alpha, j}(x):=\sum_{m=1}^{\infty} f_{\alpha, j}^{m}(x)
$$


Given any compact subset $K$ of $\Omega$, there is a positive integer $m_{0}$ such that

$$
K \cap O_{m}=\emptyset, \quad m \geq m_{0},
$$

and thus $f_{\alpha, j}$ is well defined and belongs to $\mathcal{L}_{\text {loc }}^{q}(\Omega)$. Besides, we have

$$
\left|f_{\alpha, j}\right|_{K, q} \leq \sum_{m=1}^{m_{0}}\left|f_{\alpha, j}^{m}\right|_{K, q} \leq \sum_{m=1}^{m_{0}}\left\|f_{\alpha, j}^{m}\right\|_{q}
$$

and so, if

$$
h:=\inf \left\{h_{m}: m=1,2, \ldots, m_{0}\right\}
$$

we have that

$$
\sup _{\substack{\alpha \in \mathbb{N}_{0}^{k} \\ j \in J}} h^{|\alpha|} M_{|\alpha|}\left|f_{\alpha, j}\right|_{K, q} \leq \sum_{m=1}^{m_{0}} \sup _{\substack{\alpha \in \mathbb{N}_{0}^{k} \\ j \in J}} h_{m}^{|\alpha|} M_{|\alpha|}\left\|f_{\alpha, j}^{m}\right\|_{q}<\infty .
$$

We now apply the previous theorem to obtain a bounded subset $\left\{T_{j}: j \in J\right\}$ of $\mathcal{D}_{\left(L^{p}\right)}^{\left(M_{n}\right)^{\prime}}(\Omega)$ such that

$$
\left\langle\varphi, T_{j}\right\rangle=\sum_{\alpha \in \mathbb{N}_{0}^{K}} \int_{\Omega} D^{\alpha} \varphi \cdot f_{\alpha, j} \mathrm{~d} x, \quad j \in J, \quad \varphi \in \mathcal{D}_{\left(L^{p}\right)}^{\left(M_{n}\right)}(\Omega),
$$

where the series converges absolutely and uniformly when $j$ varies in $J$ and $\varphi$ varies in any given bounded subset of $\mathcal{D}_{\left(L^{p}\right)}^{\left(M_{n}\right)}(\Omega)$.

We next choose $\varphi$ in $\mathcal{D}_{\left(L^{p}\right)}^{\left(M_{n}\right)}(\Omega)$. We find $m_{0} \in \mathbb{N}$ for which

$$
O_{m} \cap \operatorname{supp} \varphi=\emptyset, \quad m \geq m_{0} .
$$

Then

$$
\begin{aligned}
\left\langle\varphi, T_{j}\right\rangle & =\sum_{\alpha \in \mathbb{N}_{0}^{K}} \int_{\Omega} D^{\alpha} \varphi \cdot f_{\alpha, j} \mathrm{~d} x \\
& =\sum_{\alpha \in \mathbb{N}_{0}^{k}} \int_{\Omega}\left(\sum_{m=1}^{m_{0}} D^{\alpha} \varphi \cdot f_{\alpha, h j}^{m}\right) \mathrm{d} x \\
& =\sum_{m=1}^{m_{0}} \sum_{\alpha \in \mathbb{N}_{0}^{k}} \int_{\Omega} D^{\alpha} \varphi \cdot f_{\alpha, j}^{m} \mathrm{~d} x \\
& =\sum_{m=1}^{m_{0}}\left\langle\varphi, g_{m} S_{j}\right\rangle \\
& =\left\langle\sum_{m=1}^{m_{0}} \varphi \cdot g_{m}, S_{j}\right\rangle \\
& =\left\langle\varphi, S_{j}\right\rangle .
\end{aligned}
$$

Consequently, $S_{j}=T_{j}, j \in J$, and the conclusion follows.

Proposition 7. If $M_{n}, n=0,1, \ldots$ satisfies condition (1), then the canonical injection $\zeta$ from $\mathcal{D}^{\left(M_{n}\right)}(\Omega)$ into $\mathcal{D}_{\left(L^{p}\right)}^{\left(M_{n}\right)}(\Omega)$ is a topological isomorphism. 
PRoof. Clearly $\zeta$ is well defined linear and continuous. It is also plain that there exist $b>0$ and $l>0$ such that

$$
M_{n+k} \leq b l^{n} M_{n}, \quad n \in \mathbb{N}_{0} .
$$

We take now an arbitrary element $\varphi$ of $\mathcal{D}_{\left(L^{1}\right)}^{\left(M_{n}\right)}(\Omega)$ and $r \in \mathbb{N}$. Let $s$ be an integer greater that $r l$. We extend $\varphi$ to $\mathbb{R}^{k}$ such that $\varphi(x)=0, x \in \mathbb{R}^{k} \backslash \Omega$. Given $\alpha \in \mathbb{N}_{0}^{k}$ and $x \in \mathbb{R}^{k}$, we have that

$$
D^{\alpha} \varphi(x)=\int_{-\infty}^{x_{1}} \int_{-\infty}^{x_{2}} \cdots \int_{-\infty}^{x_{k}} \frac{\partial^{|\alpha|+k} \varphi(t)}{\partial^{\alpha_{1}+1} t_{1} \partial^{\alpha_{2}+1} t_{2} \ldots \partial^{\alpha_{k}+1} t_{k}} \mathrm{~d} t_{1} \mathrm{~d} t_{2} \ldots \mathrm{d} t_{k}
$$

and hence

$$
\begin{aligned}
\left|D^{\alpha} \varphi(x)\right| & \leq \int_{\Omega}\left|\frac{\partial^{|\alpha|+k} \varphi(t)}{\partial^{\alpha_{1}+1} t_{1} \partial^{\alpha_{2}+1} t_{2} \ldots \partial^{\alpha_{k}+1} t_{k}}\right| \mathrm{d} t_{1} \mathrm{~d} t_{2} \ldots \mathrm{d} t_{k} \\
& \leq\|\varphi\|_{1,1 / s} s^{-|\alpha|-k} M_{|\alpha|+k} \\
& \leq\|\varphi\|_{1,1 / s} s^{-|\alpha|-k} b l^{|\alpha|} M_{|\alpha|} \\
& \leq\|\varphi\|_{1,1 / s} b s^{-k}\left(\frac{s}{l}\right)^{-|\alpha|} M_{|\alpha|} \\
& \leq b s^{-k}\|\varphi\|_{1,1 / s} r^{-|\alpha|} M_{|\alpha|}
\end{aligned}
$$

and so

$$
\varphi \in \mathcal{D}^{\left(M_{n}\right)}(\Omega) .
$$

Thus $\zeta$ is onto. The conclusion now follows by applying a theorem of Grothendieck's, [1, p. 17].

Theorem 3. If $M_{n}, n=0,1, \ldots$ satisfies condition (1) and $\left\{S_{j}: j \in J\right\}$ is a bounded subset of $\mathcal{D}^{\left(M_{n}\right)^{\prime}}(\Omega)$, then there is, for each $j \in J$, a family $\left(f_{\alpha, j}: \alpha \in \mathbb{N}_{0}^{k}\right)$ of elements of $\mathcal{L}_{\mathrm{loc}}^{\infty}(\Omega)$ such that, given a compact subset $K$ of $\Omega$, there is $h>0$ such that

$$
\sup _{\substack{\alpha \in \mathbb{N}_{0}^{k} \\ j \in J}} h^{|\alpha|} M_{|\alpha|}\left|f_{\alpha, j}\right|_{K, \infty}<\infty
$$

and

$$
\left\langle\varphi, S_{j}\right\rangle=\sum_{\alpha \in \mathbb{N}_{0}^{k}} \int_{\Omega} D^{\alpha} \varphi \cdot f_{\alpha, j} \mathrm{~d} x, \quad j \in J, \quad \varphi \in \mathcal{D}^{‘}\left(M_{n}\right)(\Omega),
$$

where the series converges absolutely and uniformly when $j$ varies in $J$ and $\varphi$ varies in any given bounded subset of $\mathcal{D}^{\left(M_{n}\right)}(\Omega)$.

PROOF. It is an immediate consequence of the previous proposition and Theorem 2.

We put now $\mathcal{D}_{L^{p}}^{\left(M_{n}\right)}(\Omega)$ for the subspace of $\mathcal{B}_{L^{p}}^{\left(M_{n}\right)}(\Omega)$ given by the closure of $\mathcal{D}^{\left(M_{n}\right)}(\Omega)$ in that space. $\mathcal{D}_{L^{p}}^{\left(M_{n}\right)^{\prime}}(\Omega)$ will be the strong dual of $\mathcal{D}_{L^{p}}^{\left(M_{n}\right)}(\Omega)$. The two theorems that follow next are not difficult to prove by following a similar procedure to those in the proofs of Proposition 3 and Proposition 4, respectively. Those theorems constitute characterizations of certain ultradistributions of Beurling type in $\Omega$.

Theorem 4. For each $j$ in a set $J$, let $\left(f_{\alpha, j}: \alpha \in \mathbb{N}_{0}^{k}\right)$ be a family of elements of $\mathcal{L}^{q}(\Omega)$ such that there is $h>0$ with

$$
\sup _{\substack{\alpha \in \mathbb{N}_{0}^{k} \\ j \in J}} h^{|\alpha|} M_{|\alpha|}\left\|f_{\alpha, j}\right\|_{q}<\infty .
$$


Then there exists a bounded subset $\left\{S_{j}: j \in J\right\}$ of $\mathcal{D}_{L^{p}}^{\left(M_{n}\right)^{\prime}}(\Omega)$ such that

$$
\left\langle\varphi, S_{j}\right\rangle=\sum_{\alpha \in \mathbb{N}_{0}^{k}} \int_{\Omega} D^{\alpha} \varphi \cdot f_{\alpha, j} \mathrm{~d} x, \quad j \in J, \quad \varphi \in \mathcal{D}_{L^{p}}^{\left(M_{n}\right)}(\Omega),
$$

where the series converges absolutely and uniformly when $j$ varies in $J$ and $\varphi$ varies in any given bounded subset of $\mathcal{D}_{L^{p}}^{\left(M_{n}\right)}(\Omega)$.

Theorem 5. If $\left\{S_{j}: j \in J\right\}$ is a bounded subset of $\mathcal{D}_{L^{p}}^{\left(M_{n}\right)}(\Omega)$, there are $h>0$ and, for each $j \in J$, a family $\left(f_{\alpha, j}: \alpha \in \mathbb{N}_{0}^{k}\right)$ of elements of $\mathcal{L}^{q}(\Omega)$ such that

$$
\sup _{\substack{\alpha \in \mathbb{N}_{0}^{k} \\ j \in J}} h^{|\alpha|} M_{|\alpha|}\left\|f_{\alpha, j}\right\|_{q}<\infty
$$

and

$$
\left\langle\varphi, S_{j}\right\rangle=\sum_{\alpha \in \mathbb{N}_{0}^{k}} \int_{\Omega} D^{\alpha} \varphi \cdot f_{\alpha, j} \mathrm{~d} x, \quad j \in J, \quad \varphi \in \mathcal{D}_{L^{p}}^{\left(M_{n}\right)}(\Omega),
$$

where the series converges absolutely and uniformly when $j$ varies in $J$ and $\varphi$ varies in any given bounded subset of $\mathcal{D}_{L^{p}}^{\left(M_{n}\right)}(\Omega)$.

\section{Structure of the ultradistributions of Beurling type}

Given $h>0$, we put $\mathcal{E}_{0}^{\left(M_{n}\right), h}(\Omega)$ to denote the space over $\mathbb{C}$ of the complex functions $f$, defined and infinitely differentiable in $\Omega$ which vanish at infinity, as well as each of their derivatives of any order, that is, given $\epsilon>0$, and $\beta \in \mathbb{N}_{0}^{k}$, there is a compact subset $K$ in $\Omega$ for which

$$
\left|D^{\beta} f(x)\right|<\varepsilon, \quad x \in \Omega \backslash K,
$$

satisfying also that there is $C>0$, depending only on $f$, such that

$$
\left|D^{\alpha} f\right| \leq C h^{|\alpha|} M_{|\alpha|}, \quad x \in \Omega, \quad \alpha \in \mathbb{N}_{0}^{k} .
$$

We put

$$
|f|_{h}:=\sup _{\alpha \in \mathbb{N}_{0}^{k}} \sup _{x \in \Omega} \frac{D^{\alpha} f(x)}{h^{|\alpha|} M_{|\alpha|}}
$$

and assume that $\mathcal{E}_{0}^{\left(M_{n}\right), h}(\Omega)$ is endowed with the norm $|\cdot|_{h}$. We set

$$
\mathcal{E}_{0}^{\left(M_{n}\right)}(\Omega):=\bigcap_{m=1}^{\infty} \mathcal{E}_{0}^{\left(M_{n}\right), 1 / m}(\Omega)
$$

and consider $\mathcal{E}_{0}^{\left(M_{n}\right)}(\Omega)$ as the projective limit of the sequence $\left(\mathcal{E}_{0}^{\left(M_{n}\right), 1 / m}(\Omega)\right)$ of Banach spaces. $\mathcal{E}_{0}^{\left(M_{n}\right)^{\prime}}(\Omega)$ will be the strong dual of $\mathcal{E}_{0}^{\left(M_{n}\right)}(\Omega)$. By $C_{0}(\Omega)$ we represent the linear space over $\mathbb{C}$ of the complex functions $f$ defined and continuous in $\Omega$ which vanish at infinity. We put

$$
|f|_{\infty}:=\sup _{x \in \Omega}|f(x)|
$$

and assume that $C_{0}(\Omega)$ is provided with this norm.

If we replace the Banach space $X$ of Section 2 by $C_{0}(\Omega)$, following a argument similar to that of the previous section, and also using results of [4], we may obtain the next two theorems, which are a generalization of result b). 
Theorem 6. For each $j$ in a set $J$, let $\left(u_{\alpha, j} . \alpha \in \mathbb{N}_{0}^{k}\right)$ be a family of Radon measures in $\Omega$. If, given an arbitrary compact subset $K$ of $\Omega$, there is $h>0$ such that

$$
\sup _{\substack{\alpha \in \mathbb{N}_{0}^{k} \\ j \in J}} h^{|\alpha|} M_{|\alpha|}\left\|u_{\alpha, j}\right\|(K)<\infty,
$$

then there exists a bounded subset $\left\{S_{j}: j \in J\right\}$ of $\mathcal{D}^{\left(M_{n}\right)^{\prime}}(\Omega)$ such that

$$
\left\langle\varphi, S_{j}\right\rangle=\sum_{\alpha \in \mathbb{N}_{0}^{k}}\left\langle D^{\alpha} \varphi, u_{\alpha, j}\right\rangle, \quad j \in J, \quad \varphi \in \mathcal{D}^{\left(M_{n}\right)}(\Omega),
$$

where the series converges absolutely and uniformly when $j$ varies in $J$ and $\varphi$ varies in any given bounded subset of $\mathcal{D}^{\left(M_{n}\right)}(\Omega)$.

Theorem 7. If $\left\{S_{j}: j \in J\right\}$ is a bounded subset of $\mathcal{D}^{\left(M_{n}\right)^{\prime}}(\Omega)$, there is, for each $j \in J$, a family $\left(u_{\alpha, j}: \alpha \in \mathbb{N}_{0}^{k}\right)$ of Radon measures in $\Omega$ such that, given a compact subset $K$ of $\Omega$, there is $h>0$ with

$$
\sup _{\substack{\alpha \in \mathbb{N}_{0}^{k} \\ j \in J}} h^{|\alpha|} M_{|\alpha|}\left\|u_{\alpha, j}\right\|(K)<\infty
$$

and

$$
\left\langle\varphi, S_{j}\right\rangle=\sum_{\alpha \in \mathbb{N}_{0}^{k}}\left\langle D^{\alpha} \varphi, u_{\alpha, j}\right\rangle, \quad j \in J, \quad \varphi \in \mathcal{D}^{\left(M_{n}\right)}(\Omega),
$$

where the series converges absolutely and uniformly when $j$ varies in $J$ and $\varphi$ varies in any given bounded subset of $\mathcal{D}^{\left(M_{n}\right)}(\Omega)$.

We put $\mathcal{E}^{\left(\stackrel{\circ}{n}_{n}\right)}(\Omega)$ for the subspace of $\mathcal{E}_{0}^{\left(M_{n}\right)}(\Omega)$ given by the closure of $\mathcal{D}^{\left(M_{n}\right)}(\Omega) . \mathcal{E}^{\left(\stackrel{\circ}{n}_{n}\right) \prime}(\Omega)$ will denote its strong dual. The elements of this last space may be considered as Beurling ultradistributions in $\Omega$. We characterize those ultradistributions in the following two theorems. Their proofs may be obtained by conveniently adapting the proofs of Proposition 3 and Proposition 4, respectively.

Theorem 8. For each $j$ in a set $J$, let $\left(\mu_{\alpha, j} . \alpha \in \mathbb{N}_{0}^{k}\right)$ be a family of complex Borel measures in $\Omega$ such that there is $h>0$ with

$$
\sup _{\substack{\alpha \in \mathbb{N}_{0}^{k} \\ j \in J}} h^{|\alpha|} M_{|\alpha|}\left|\mu_{\alpha, j}\right|(\Omega)<\infty .
$$

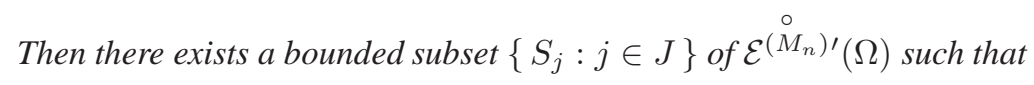

$$
\left\langle\varphi, S_{j}\right\rangle=\sum_{\alpha \in \mathbb{N}_{0}^{k}} \int_{\Omega} D^{\alpha} \varphi \cdot \mathrm{d} \mu_{\alpha, j}, \quad j \in J, \quad \varphi \in \mathcal{E}^{\left(M_{n}\right)}(\Omega),
$$

where the series converges absolutely and uniformly when $j$ varies in $J$ and $\varphi$ varies in any given bounded subset of $\mathcal{E}^{\left(M_{n}\right)}(\Omega)$.

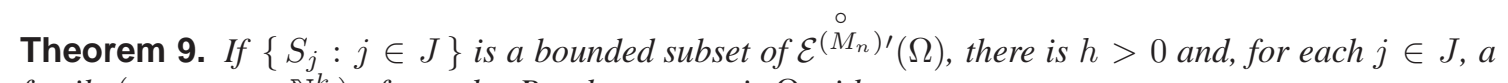
family $\left(\mu_{\alpha, j}: \alpha \in \mathbb{N}_{0}^{k}\right)$ of complex Borel measures in $\Omega$ with

$$
\sup _{\substack{\alpha \in \mathbb{N}_{0}^{k} \\ j \in J}} h^{|\alpha|} M_{|\alpha|}\left|\mu_{\alpha, j}\right|(\Omega)<\infty,
$$


and

$$
\left\langle\varphi, S_{j}\right\rangle=\sum_{\alpha \in \mathbb{N}_{0}^{k}} \int_{\Omega} D^{\alpha} \varphi \cdot \mathrm{d} \mu_{\alpha, j}, \quad j \in J, \quad \varphi \in \mathcal{E}^{\left(\stackrel{\circ}{n}_{n}\right)}(\Omega),
$$

where the series converges absolutely and uniformly when $j$ varies in $J$ and $\varphi$ varies in any given bounded subset of $\mathcal{E}^{\left(\stackrel{\circ}{M_{n}}\right)}(\Omega)$.

Acknowledgement. The author has been partially supported by MEC and FEDER Project MTM200803210 .

\section{References}

[1] Grothendieck, A., (1955). Produits Tensoriels Topologiques et Espaces Nucléaires, Memoirs of the American Mathematical Society, 16.

[2] Komatsu, H., (1973). Ultradistributions I. Structure theorems and characterizations, J. Fac. Sci. Uni. Tokyo, 20, 25-105.

[3] Schwartz, L., (1966). Théorie des Distributions, Hermann, Paris.

[4] VAldivia, M., (2008). On the structure of the ultradistributions of Beurling type, RACSAM, Rev. R. Acad. Cien. Serie A. Mat., 102, 221-235.

Manuel Valdivia

Departamento de Análisis Matemático, Facultad de Matemáticas

Universidad de Valencia

Calle Doctor Moliner, 50

46100 Burjassot (Valencia, Spain). 